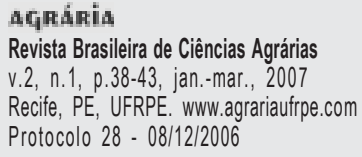

Alexander R. M. Sablowski ${ }^{1}$

Joaquim C. Gonçalez ${ }^{2}$

José E. I. Encinas ${ }^{2}$

Armando Caldeira-Pires ${ }^{3}$

Fernando N. Gouveia ${ }^{1}$

Raoni Carneiro ${ }^{4}$

\section{Avaliação da cadeia produtiva madeiro-moveleiro no Distrito Federal utilizando a análise de fluxo de substância}

\author{
RESUMO
}

No diagnóstico da cadeia madeiro-moveleiro no Distrito Federal observou-se a origem da matériaprima, a linha de produção, principais insumos, destino final dos produtos e resíduos gerados. Na elaboração do diagnóstico, utilizou-se a ferramenta "análise de fluxo de substância", que separou a madeira processada na forma serrada e em painéis reconstituídos, e seus resíduos (serragem e aparas) a substância avaliada. Os dados primários coletados em 43 empresas, localizadas no Distrito Federal, foram processados e analisados pelo programa "Programa 5 menos que são mais - redução de desperdício" do Sebrae - DF. Duas linhas de produção foram identificadas, uma constituída por madeireiras que fornecem a madeira serrada, e marcenarias produtoras de portais e janelas para construção civil. A outra foi o pólo moveleiro em desenvolvimento no Distrito Federal. A primeira foi responsável pela maior parte dos resíduos gerados em função do volume de substância madeira utilizada, emprego de maquinários antigos e a qualificação da mão-de-obra. A segunda linha produziu menor quantidade de resíduo devido à otimização da produção, gerando a melhoria no rendimento associada a ganhos no comportamento ambiental da cadeia como um todo.

Palavras-chave: ecologia industrial, processamento de madeira, gestão ambiental

\section{Evaluation of the chain of production of the wood products sector in the Federal District using substance flow analysis}

\section{ABSTRACT}

A diagnosis is made of the chain of production of the wood products sector in the Federal District, including the origin of the raw material, existing lines of production, main inputs, final destination of the products, main types of residues, and residue disposal. The Substance Flow Analysis tool was used in the diagnosis, where the substance evaluated was lumber, divided into composition boards and residues (fine and and coarse residues). The primary data were collected in 43 companies located in the Federal District and subsequently processed and analyzed using the Sebrae - DF software "Five Less which are More". Two distinct lines of production were identified: firstly, the sawmills that supply lumber and carpentry workshops that produce doors and windows for the civil construction industry; and secondly, the cabinetmaking hub being established in the Federal District. Most residues came from the first line of production because of the large volume of sawlogs processed, outmoded machinery used and low levels of skill of the workers. The second line of production had less waste, as a result of the optimization of production through productivity improvements associated with gains in the environmental behavior of the production chain as a whole.

Key words: industrial ecology, wood processing, environmental management 


\section{INTRODUÇÃO}

O setor florestal representa 4,5\% do Produto Interno Bruto nacional. Ocupa posição estratégica quanto aos desenvolvimentos sociais e tecnológicos do Brasil, tendo as empresas do setor contribuído sobremaneira com o crescimento econômico do país nos últimos anos. A cadeia produtiva da madeira em 2003 exportou U\$ 2,89 bilhões (Abimci, 2004).

Entretanto, o conhecimento sobre a cadeia produtiva e os diferentes segmentos que integram esse setor ainda é incompleto, sob o ponto de vista ambiental e produtivo. Identificase que a avaliação integrada destes, buscando a sustentabilidade econômica e ambiental da cadeia é necessária para a aceitação dos produtos gerados no mercado nacional e internacional.

Para avaliação do comportamento ambiental e de produção, atualmente se destacam-se as ferramentas baseadas no conceito da Ecologia Industrial como ficou demonstrado nos trabalhos de Lifset (1998), Blum et al.(1998), Sablowski \& Vale (2002) e Sablowski et al.(2004).

De acordo com Hüttler \& Kowalski (1998), a análise de fluxo material está se transformando em uma ferramenta poderosa para descrever e analisar o desenvolvimento sustentável em um contexto macro.

Segundo Bherensmeyer et al. (1998), toda análise é baseada na definição de uma unidade funcional que descreva a entidade operacional a que todos os cálculos são relacionados. Para análise do fluxo de materiais dentro do metabolismo industrial, existem diferentes modelos, dentre os quais se destacam a "análise do fluxo de substância", a "análise do ciclo de vida" e a "análise do fluxo de massa e energia".

De acordo com o documento "Perfil competitivo do Distrito Federal" (Serviço de Apoio às Micro e Pequenas Empresas no Distrito Federal, Sebrae-DF, 2003), existem 596 empresas legalmente constituídas dentro da cadeia madeiro-moveleiro no Distrito Federal.

Del Menezzi \& Bounduelle (2002) avaliaram 10\% das indústrias de madeira e mobiliário do DF e constataram que, apesar de as empresas terem conhecimento sobre a certificação de qualidade, até 2001 nenhuma delas havia obtido certificado pertinente, possivelmente pelo fato dos consumidores serem pouco exigentes quanto aos produtos certificados. Outrossim, a maioria das empresas afirmou praticar controle de qualidade em suas linhas de produção, embora o método utilizado não fosse totalmente claro.

Em um estudo desenvolvido pelo Núcleo de Gestão Ambiental do Sebrae-DF, em 58 empresas, constatou-se a demanda por programas de certificação ambiental neste setor (SebraeDF, 2005).

A carência de informações e a falta de uma política clara para os segmentos de madeira e móveis no Distrito Federal relegam as empresas a um patamar de pouca relevância no contexto econômico local, regional e nacional. Esta falta de incentivo contribui significativamente para o não-aperfeiçoamento da cadeia produtiva como um todo.

Neste trabalho, utilizou-se especificamente a "análise de fluxo de substância", por meio da qual se avalia uma determinada substância, suas entradas e saídas em processos eco- nômicos ou naturais de um sistema espacial e temporal, neste caso constituídos pelas empresas do pólo moveleiro do Distrito Federal. Na avaliação, considerou-se o comportamento ambiental e de produção.

\section{MATERIAL E MÉTODOS}

A coleta de dados primários foi executada através do "Programa 5 menos que são mais - redução de desperdício" do Sebrae-DF, que utiliza um questionário estruturado e tem como objetivos:

- Avaliar a origem da matéria prima (atacado, varejo e indústria) e quantificá-la;

- Identificar os principais segmentos existentes dentro da cadeia produtiva;

- Identificar, quantificar e determinar o destino dos resíduos gerados;

- Observar os insumos utilizados;

- Avaliar o comportamento ambiental associado ao comportamento de produção;

- Identificar os pontos críticos que permitam a redução do desperdício, com conseqüente diminuição da produção de resíduos e consumo de energia e água, através de um programa adequado de gestão ambiental.

Os dados primários foram utilizados para elaborar o fluxograma da substância madeira na cadeia produtiva madeiromoveleiro do Distrito Federal (Figura 1). Como entrada do sistema, foi considerada a origem geográfica e comercial do material madeira; e como saída do sistema, o local de descarte ou reuso dos resíduos de madeira. Ao longo do sistema, foram identificadas como entradas secundárias os insumos utilizados; e como saídas secundárias, o descarte de resíduos de insumos e emissões. As entradas e saídas secundárias foram apresentadas em fluxograma correspondente sem distinção da quantidade.

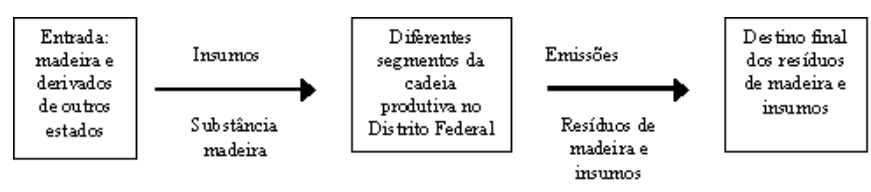

Figura 1. Estrutura do fluxograma da substância madeira e insumos no diagnóstico da cadeia produtiva madeiro-moveleiro do Distrito Federal

Figure 1. Structure of the wood and inputs flowgraph for the diagnosis of the chain of production of the wood products sector in the Federal District

O levantamento foi executado em 43 empresas, dentro de um universo de 596 legalmente constituídas (Sebrae-DF, 2003). As empresas foram escolhidas através de "amostragem estratificada", na qual os estratos representaram os diferentes pólos industriais (seis regiões), e a intensidade amostral variou de acordo com a região em função do número de empresas existentes. As regiões amostradas foram: Guará, Taguatinga, Vicente Pires, Ceilândia, Samambaia e Brasília.

$\mathrm{O}$ material de entrada foi dividido em dois grupos, em relação a sua composição: madeira serrada e madeira trans- 
formada em painéis: MDF (Medium Density Fiberboard), compensado, aglomerado e laminado. Os resíduos (saída) ficaram diferenciados em serragem e aparas (aparas são os resíduos de madeira provenientes do desdobro da madeira e corte de painéis com exceção para a serragem). O volume da substância madeira foi diferenciado por segmento da cadeia, assim como os resíduos gerados em relação a sua origem e destino. Para o cálculo da madeira serrada, utilizou-se o volume $\left(\mathrm{m}^{3}\right)$ fornecido pela empresa avaliada. Para a madeira reconstituí$\mathrm{da}$, efetuou-se a estimativa do volume $\mathrm{em}^{3}$, de acordo com as dimensões médias dos diferentes painéis e a respectiva quantidade média de unidades utilizadas pela empresa. As dimensões médias por tipo de painel são apresentadas na Tabela 1.

Tabela 1. Dimensão média por tipo de painel

Table 1. Mean dimension for wood product type

\begin{tabular}{cc}
\hline Tipo & Dimensão média $(\mathrm{m})$ \\
\hline Compensado & $1,60 \times 2,20 \times 0,015$ \\
Aglomerado & $2,75 \times 1,83 \times 0,015$ \\
MDF & $2,75 \times 1,83 \times 0,015$ \\
Laminado & $1,0 \times 1,0 \times 0,001$ \\
\hline Fonte: Lammy (2005) &
\end{tabular}

Para a estimativa dos volumes totais e a representatividade por segmento existente no DF, considerou-se a média obtida pelas 43 empresas avaliadas, e extrapolou-se para o total de empresas legalmente constituídas e existentes no Distrito Federal.

O fluxograma de insumos, ao longo da cadeia produtiva, considera os insumos existentes como entrada, e os resíduos oriundos e suas correspondentes emissões como saída. Ressalta-se que, como já mencionado neste caso, os volumes de entrada e saída não foram quantificados.

$\mathrm{Na}$ análise dos fluxogramas, foram identificados os pontos críticos cuja alteração poderá viabilizar a melhoria do comportamento ambiental da cadeia como um todo em relação aos resíduos gerados e sistema de gestão da linha de produção.

\section{RESULTADOS E DISCUSSÃO}

A cadeia produtiva de madeira no Distrito Federal é composta por cinco segmentos diferenciados em:

- Comércio: composto por representantes de indústrias de outros estados, serrarias e empresas de corte de painéis reconstituídos. A madeira serrada apresenta maior fluxo em relação aos painéis de madeira;

- Construção civil: composta por indústrias manufaturadoras de peças destinadas ao uso na construção civil (estrutura ou acabamento), podendo ser as mesmas personalizadas ou padronizadas. $\mathrm{O}$ uso de madeira serrada predomina sobre o uso de painéis reconstituídos;

- Divisórias: indústria de divisórias, estações de trabalho e móveis para escritório (pouco representativo). Neste tipo de indústria, o consumo de madeira reconstituída é muito superior em relação ao de madeira serrada;
- Indústria de móveis: produção de móveis em série ou personalizados para uso doméstico ou em escritórios e instalações comerciais. Divide-se em um subsegmento que utiliza somente painéis reconstituídos (padronizada) e o subsegmento de móveis de projeto específico;

- Instalações comerciais: responsável pela manufaturação de móveis específicos para instalações comerciais do tipo armazenagem e conservação. Neste segmento, os fluxos de painéis reconstituídos e madeira serrada são equivalentes.

A madeira processada em serraria tem como origem principal o estado do Pará, e a madeira transformada em painéis tem como origem principal os estados do Mato Grosso, São Paulo e Paraná.

\section{Fluxo da substância madeira no Distrito Federal}

O Distrito Federal caracteriza-se por ser uma região de grande consumo de matéria-prima florestal na forma de madeira processada (aproximadamente 22.142,92 $\mathrm{m}^{3}$ por mês entrada no sistema demonstrado na Figura 2). Os estados do Pará e Rondônia são os responsáveis pelo fornecimento de madeira processada na forma serrada. Já os estados de São Paulo, Mato Grosso e Paraná fornecem a madeira reconstituída (compensado, MDF e aglomerado).

Os principais consumidores de madeira no Distrito Federal são os segmentos da construção civil e indústria moveleiro, com $78,26 \%$ e $16,51 \%$ do total, respectivamente (madeira serrada e reconstituída).

O fluxo da substância madeira da cadeia madeiro-moveleiro do Distrito Federal (Figura 1) apresenta valores de entrada no sistema não-equivalentes aos totais de distribuição nos cinco segmentos. Isto se deve à existência de estoque com grande flexibilidade no segmento comércio, que está sob influência direta do mercado. Como exemplo, observou-se que durante o levantamento dos dados primários, o segmento comércio absorveu do sistema $23,78 \mathrm{~m}^{3}$ de madeira reconstituída e $88,00 \mathrm{~m}^{3}$ de madeira serrada, mas liberou para o sistema $43,90 \mathrm{~m}^{3}$ de madeira reconstituída e $2,19 \mathrm{~m}^{3}$ de madeira serrada. Ou seja, durante o período de levantamento, o segmento de comércio liberou parte do estoque de madeira reconstituída já existente e reteve praticamente toda a madeira serrada adquirida. Conseqüentemente, o total de entrada no sistema durante o período é diferente do total distribuído nos segmentos. Esta diferença deve ser incluída no cálculo da média mensal total para a execução da análise. Ao calcular a representatividade de um determinado segmento da cadeia em relação ao total, deve ser considerado positivamente a quantidade de substância que ingressa no sistema com origem no estoque do segmento comércio, e de forma negativa a quantidade que fica retida em seu estoque, alterando a quantidade total que ingressa no sistema como um todo.

Na Tabela 2 é diferenciado percentualmente o consumo por tipo de madeira (serrada ou painéis reconstituídos) dos segmentos indústria moveleiro e construção civil (maiores consumidores) em relação ao sistema como um todo.

A distribuição do consumo médio em volume de madeira processada na forma serrada e painel, por setor da cadeia produtiva de madeira do DF, é apresentado na Tabela 3. Observou-se que os valores totais não correspondem aos valo- 


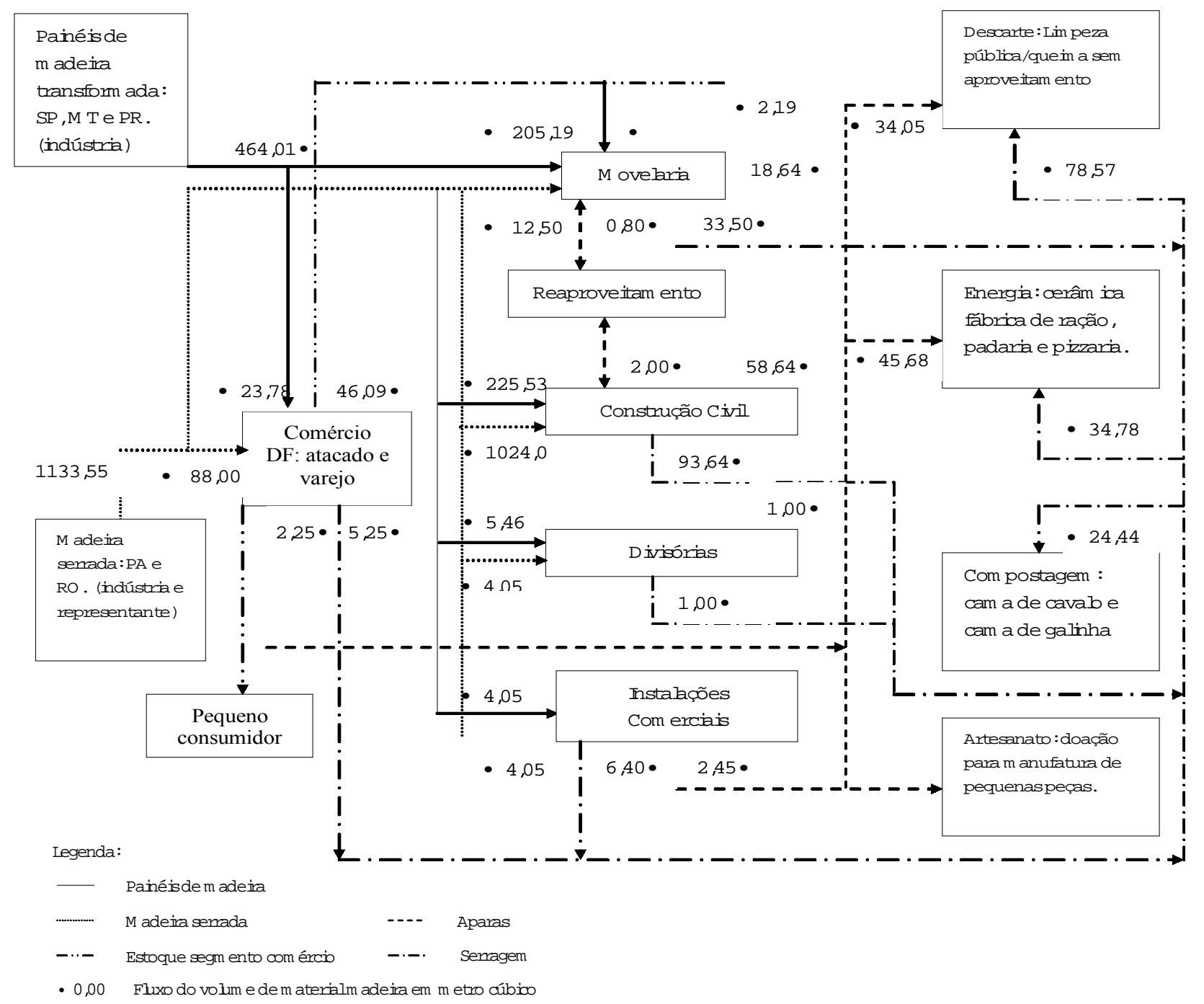

Figura 2. Fluxograma da substância madeira na cadeia madeiro-moveleiro do Distrito Federal - DF em volume $\left(\mathrm{m}^{3}\right)$

Figure 2. Fluxogram of wood in the wood products chain in the Federal District by volume $\left(\mathrm{m}^{3}\right)$

Tabela 2. Principais consumidores por tipo de madeira processada no Distrito Federal em percentual

Table 2. Main consumers of different types of processed wood in the Federal District

\begin{tabular}{ccc}
\hline \multirow{2}{*}{ Segmento } & \multicolumn{2}{c}{ Madeira processada (\%) } \\
\cline { 2 - 3 } & Serrada & Painel \\
\hline Indústria moveleira & 1,40 & 51,21 \\
Construção civil & 97,73 & 46,37 \\
Outros segmentos & 0,87 & 2,42 \\
\hline
\end{tabular}

res de entrada no sistema devido à inclusão dos valores estocados e liberados pelo segmento de comércio.

O setor de construção civil é alimentado por empresas fornecedoras de portas, janelas, batentes, portais e, principalmente, madeiramento para estrutura de telhado. Esse perfil está de acordo com o intenso movimento de expansão demográfica iniciada na última década do século XX no Distrito Federal.
Tabela 3. Consumo médio mensal de madeira processada em volume $\left(\mathrm{m}^{3}\right)$ por setor da cadeia produtiva de madeira do Distrito Federal

Table 3. Average monthly consumption of processed wood by volume $\left(\mathrm{m}^{3}\right)$, by sector of the wood chain of production in the Federal District

\begin{tabular}{lrr}
\hline \multirow{2}{*}{ Segmento } & \multicolumn{2}{c}{ Madeira Processada $\left(\mathbf{m}^{3}\right)$} \\
\cline { 2 - 3 } & Serrada & Painel \\
\hline Comércio & $1.219,72$ & 329,60 \\
Construção Civil & $14.193,12$ & $3.125,95$ \\
Divisórias & 69,30 & 75,68 \\
Instalações comerciais & 56,13 & 56,14 \\
Indústria moveleira & 203,61 & $3.452,50$ \\
Total & $15.741,88$ & $7.039,87$ \\
\hline
\end{tabular}

O setor moveleiro está dividido em dois segmentos. O primeiro tem como atividade principal a produção em série de móveis de constituídos de MDF (Medium Density Fiberboard), e o segundo é composto por marcenarias e pequenas indústrias moveleiras que efetuam projetos sob encomenda 
que utilizam, além de madeiras transformada em painéis, também a madeira serrada.

Do total da substância madeira, 14,18\% ficou transformada em resíduos, sendo $1.188,95 \mathrm{~m}^{3}$ na forma de aparas e $1.951,41 \mathrm{~m}^{3}$ na forma de serragem (Tabela 4 ), concluindo-se que $49,71 \%$ dos resíduos são descartados sem nenhum aproveitamento e que a utilização para energia se destaca dentre as formas de reutilização destes resíduos em curto prazo no Distrito Federal.

Tabela 4. Destinação dos resíduos da cadeia madeiro-moveleiro do Distrito Federal

Table 4. Disposal of wood residues from the chain of production of the wood products sector in the Federal District

\begin{tabular}{lrr}
\hline \multirow{2}{*}{ Destinação } & \multicolumn{2}{c}{ Resíduos $\left(\mathrm{m}^{3}\right)$} \\
\cline { 2 - 3 } & Serragem & Aparas \\
\hline Limpeza pública & $1.089,02$ & 471,95 \\
Energia & 482,07 & 633,15 \\
Compostagem & 380,33 & 0,00 \\
Artesanato & 0,00 & 45,05 \\
Reaproveitamento & 0,00 & 38,81 \\
& $1.951,41$ & $1.188,95$ \\
\hline
\end{tabular}

Ressalta-se que o volume total de aparas com destino ao reaproveitamento ou abastecimento de pequenas indústrias artesanais $\left(38,81 \mathrm{~m}^{3}\right.$ por mês) é extremamente baixo em relação ao total gerado que é de $1.188,95 \mathrm{~m}^{3}$. E esta é uma utilização mais nobre e passível de ser executada em médio e curto prazos.

No caso da serragem especificamente, $19,50 \%$ do material está sendo usado para a formação de "cama de galinha ou cavalo" e, posteriormente, utilizada para compostagem. Esta utilização possui um limitante: a necessidade de a serragem ser de origem do corte de madeira serrada, pois a madeira reconstituída na forma de painéis possui compostos químicos que podem ser prejudiciais tanto para os animais (forro de estábulo ou granja), como para a utilização em composto para adubação de vegetais para consumo na forma de alimento.

\section{Fluxo de insumos}

No fluxo dos principais insumos existentes ao longo da cadeia madeiro-moveleiro do Distrito Federal (Figura 3) foram considerados também os resíduos conseqüentes e emissões na forma de calor e ruído, sem a contabilização de suas respectivas quantidades.

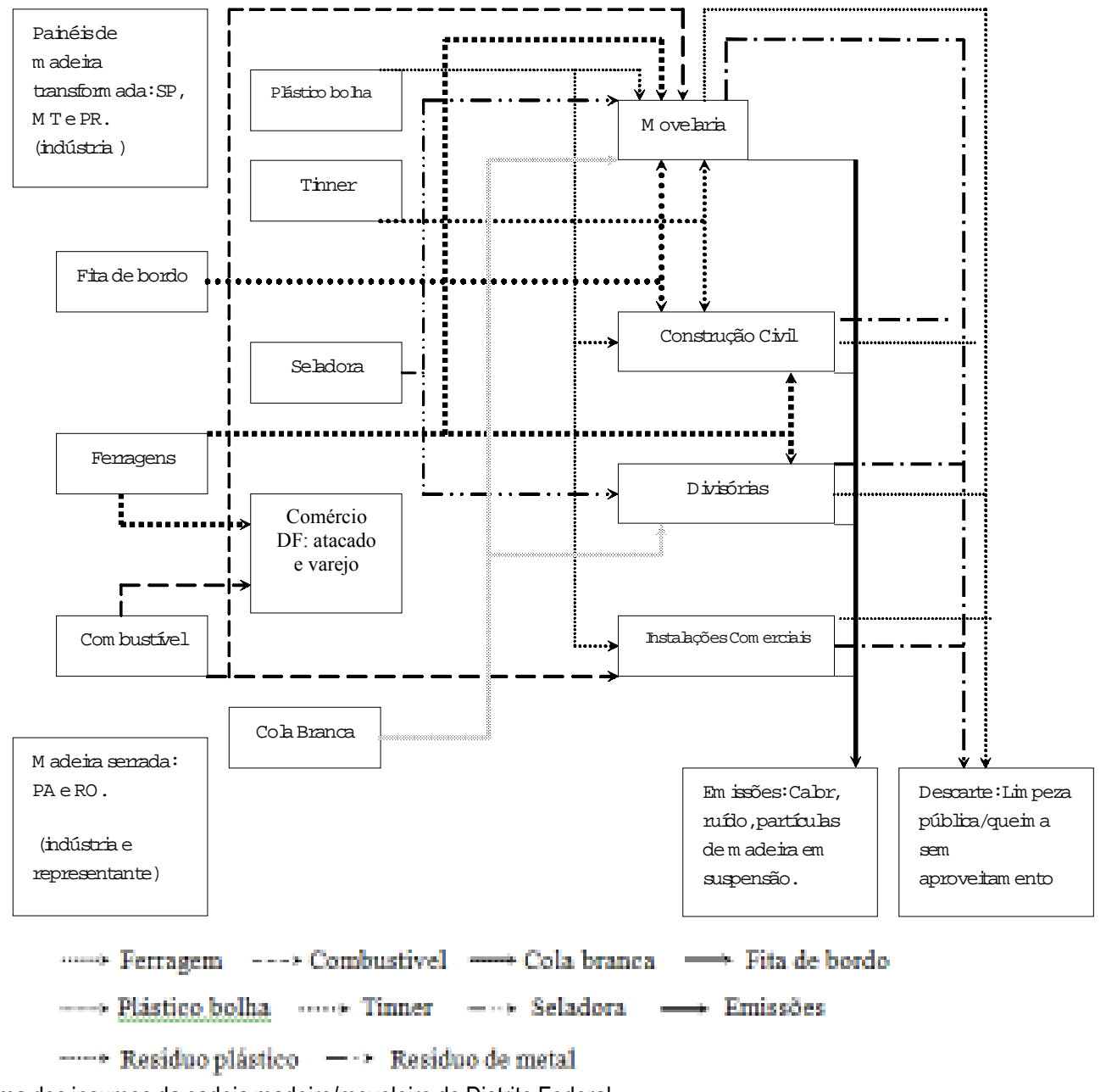

Figura 3. Fluxograma dos insumos da cadeia madeiro/moveleiro do Distrito Federal

Figure 3. Input flowgraph of the chain of production of wood products sector in the Federal District 
A cadeia produtiva de madeira no Distrito Federal é, hoje, composta por dois segmentos principais, quais sejam, o da construção civil e o da indústria moveleira.

O primeiro é representado pelas empresas associadas ao setor da construção civil, sendo responsável pela maior parte do consumo de madeira no DF. É um setor que pouco se desenvolveu em tecnologia, fato percebido ao se observar os maquinários utilizados que, em sua maioria são antigos, e responsáveis em parte pela grande geração de resíduos existentes, entretanto o principal problema é o padrão de dimensionamento das peças que abastecem a cadeia produtiva. Esse fato não anula a necessidade dos empresários se conscientizarem da importância de melhorar suas próprias empresas na busca de alternativas para a minimização das perdas.

São necessárias ações voltadas à criação de novas linhas de produção capazes de absorver as peças de menor dimensão, bem como estimular a criação de parcerias entre as empresas do setor, seja para revenda da matéria-prima, seja para a criação de novas linhas de produção.

O segundo é representado pela indústria moveleira. É um segmento que vem se estabelecendo como pólo industrial no Distrito Federal. O principal problema encontrado neste setor tem sido a falta de mão-de-obra especializada e o padrão da matéria-prima industrial fornecida. Entretanto, como ponto positivo, pode-se citar a consciência dos empresários do setor quanto a uma boa administração de suas linhas de produção para redução do desperdício e de sua responsabilidade com o meio ambiente. Outro ponto importante é o descarte de embalagens com resíduos tóxicos, insumos necessários à linha de produção desse setor.

Em relação aos resíduos gerados, são necessárias, conforme constatamos, ações voltadas à criação de novas linhas de produção capazes de absorver as peças de menor dimensão, bem como estimular a criação de parcerias entre as empresas do setor, seja para revenda da matéria-prima, seja para a criação de novas linhas de produção.

\section{CONCLUSÕES}

A cadeia produtiva de madeira no Distrito Federal hoje é composta por dois segmentos principais, da construção civil e da indústria moveleiro.

O primeiro é responsável pela maior parte do consumo de madeira no DF e é caracterizado por baixo desenvolvimento em tecnologia, principal fato responsável pela geração de resíduos que associado ao padrão de dimensionamento das peças que abastecem a cadeia produtiva, representam o fator de baixa eficiência do setor em relação ao uso de materiais.

O segundo é representado pela indústria moveleiro. É um segmento que vem se estabelecendo como pólo industrial no Distrito Federal, onde o principal problema encontrado foi a falta de mão-de-obra especializada e o padrão da matéria-prima industrial fornecida. Devendo-se também atentar ao descar- te de embalagens com resíduos tóxicos, insumos necessários à linha de produção desse setor.

Conclui-se que são necessárias ações voltadas à criação de novas linhas de produção capazes de absorver as peças de menor dimensão, bem como estimular a criação de parcerias entre as empresas do setor direcionado à utilização dos resíduos, o que tecnicamente é viável.

\section{LITERATURA CITADA}

ABIMCI - Associação Brasileira da Indústria de Madeira Processada. Base florestal é o segundo maior exportador. $<$ http://www.abimci.com.br> Acesso em 21 jun. 2004.

Behrensmeyer, R.; Bringesu, S.; Schütz H. Material flow accounts part I - General aspects, aluminium, national overall accounts. Luxemburgo: Wuppertal Institute, 1998, 94 p. Blum, L.; Denison, R. A.; Ruston, J. F.. A life-cycle approach to purchasing and using environmentally preferable paper: A summary of paper task force report. Journal of Industrial Ecology, New Haven, v.1, n.3, p.15-46, 1998.

Del Menezzi, C.S.; Bonduelle, G.M.. Diagnóstico do controle de qualidade na indústria da madeira e do mobiliário no Distrito Federal. In: Congresso Ibero-Americano de Pesquisa e Desenvolvimento de Produtos Florestais, 2, Seminário em Tecnologia da Madeira e Produtos Florestais Não-madeiráveis, 1, 2002. Curitiba. Anais... Curitiba: UFPR, 2002. $19 \mathrm{p}$.

Hüttler, W.; Kowalski, M.F.. Society`s metabolism - The state of the art - The intellectual history of material flow analysis, part II. Vienna: University of Vienna, 1998. p.31.

Lammy Industrial Madeireira da Amazônia - Lammy. Pisos de madeira e compensados no Brasil. Disponível em $<$ www.lammy.com.br>. Acesso em: 25 fev. 2005.

Lifset, R. Examining the industrial ecology of a renewable resource. Journal of Industrial Ecology, New Haven, v.1, n.3, p.1-2, 1998.

Sablowski, A. R. M. ; Vale, A. T. . Fluxo de massa e energia associada ao sistema de gestão ambiental no controle da eficiência na industria madeireira. In: $8^{\circ}$ congresso florestal brasileiro, 2003, São Paulo. 8, São Paulo : Sbef, 2003. v. 1. p. $139-151$.

Sablowski, A. R. M. ; Vale, A. T.; Martins, I. S. . Análise da eficiência de produção em uma indústria de laminados e compensados. In: Simpósio Brasileiro de Pós Graduação em Engenharia Florestal, 3, 2004, Manaus. Inpa, 2004. v. 1. p. 142-144.

SEBRAE - DF. Serviço de Apoio às Micro e Pequenas Empresas no Distrito Federal - Sebrae/DF Perfil competitivo do Distrito Federal. Brasília: Sebrae-DF, 2003. 260 p.

SEBRAE - DF. Serviço de Apoio às Micro e Pequenas Empresas no Distrito Federal. Consolidação da cadeia madeiromoveleiro do DF. Brasília: Sebrae-DF - Núcleo de Gestão Ambiental, 2005. 165p. 